\title{
Kinematic Analysis of the whole body Center of Gravity Irajectory and Time Structure of the Tennis Serve
} Performanee.

\author{
Sohair Talaat Ebrahim Ellabany \\ Department of Sport Training and Movement Sciences, Faculty of Physical Education for Girls, Alexandria University, Egypt. \\ Mansour Abdel-Hamid lsmail Attaallah \\ Department of Physical Education Foundation, Faculty of Sport Education for Men, Alexandria University, Egypt.
}

\begin{abstract}
The purpose of this study was to examine the trajectory of the Center of gravity and hitting hand motion, how the momentum and force transfer from the whole body to the ball. High level player (age 32 ; height $181 \mathrm{~cm} ; \mathrm{mass} 75 \mathrm{~kg}$ ) volunteered in this study. After the player warm up he had five trials before recording. Six trials were captured using two synchronize camera (Basler $100 \mathrm{~Hz}$ ). The best successful three trials were selected for $3 D$ motion analysis using (Winanalyze v2.0). The force curve for Center of gravity and hitting hand had a same path but there is a little difference in the momentum curve at the impact instant. This study suggests that combining of balance and coordination exercises may help to maintain the Center of gravity force trajectory in the same path with the hitting hand which gives an advantage for success serve. The takeoff within minimum time helps to increase the velocity of the Center of gravity and doesn't loss a generated force from the body to the hitting hand.
\end{abstract}

\section{Introduction:}

$\mathrm{T}$ The serve is the only stroke in tennis that is $100 \%$ under control of the player, and it is not a response to a ball hit by an opponent. It is also a highly complex skill because of its reliance on multiple segments in the kinetic chain to produce power through properly timed rotations and complex coordinated muscular activations. as well as the most important from a strategic standpoint(Kovacs, Chandler, \& Chandler, 2007) The difficulty in the movement results from the summation of forces from the ground up through the kinetic chain and out into the ball.

The serve is one of the most important skills a tennis player must acquire in order to have an effective attack. The primary objective of the serve is to direct the ball into the service area on the opponents side of the court. It is also one of the most difficult skills to execute as the act of throwing the ball and then hitting it on its downward flight, requires a complex multi segment co-ordination between the ball, the moving body segments, the trunk and the lower limbs.(R. Bahamonde, 2000).Tennis serve can generally be divided into three patterns: flat, spin, and slice. Tennis serve transfers the GRF to the trunk by tiptoeing of the lower extremity, utilizing an effective integrity of body segments and the continuity of limb motion from proximal end to distal end(Kuo-ChengLo, 2004).The Center of gravity has a great importance in connection with the 'follow through phase' of the serve.
The preparation phase results in the storing of potential energy that can be utilized as kinetic energy during the acceleration phase. In an efficiently functioning kinetic chain, the legs and trunk segments are the engine for the development of force and the stable proximal base for distal mobility(M. S. Kovacs \& T. S. Ellenbecker, 2011)The serve allows players to shift their body weight forward in the direction of the ball. This allows the server to produce more power and momentum due to them shifting their body weight forward allowing connection with the ball and allowing a smoother follow through. This can be linked to the different aspects of the kinetic chain. However, it is important not to over balance during the serve as the serve is not the only aspect of the tennis game that needs to be focused on. It is important to ensure that the server can remain stable and not over balance as this could affect how quickly and easily to move to reach to the next ball that is returned over the net.(Blazevich, 2012; De Subijana \& Navarro, 2010) The movement will be efficient if it was performed, in the biomechanical sense, correctly, and if possible optimally (Knudson \& Morrison, 2000).The efficient serve will be performed only when a player, in a movement that is biomechanical correct, connects a large amount of force in less time, or in other words acceleration, which will consequently transmit to the tennis ball. Only a strong, but at the same time fast player can learn to serve efficiently(Cauraugh, 1990).The concept connected with the behavior of the Center of gravity and balance is necessary to understand the correct serve. Bad balance or loss of balance is one of the most 
common mistakes when serving(Bruce Elliott, 2003). Temporal or phase analysis plays important role in modern sport biomechanics. It is the most versatile biomechanical method of analysis across different sports, because it is based on time only and can represent different motions as a sequence of phases and sub-phases(Kleshnev, 2007). The phase analysis can play integrating role for other biomechanical methods, such as kinematics and kinetics analysis using video or instrumentation measurements. It can decrease complexity of many sporting techniques and provide a better understanding to coaches and, which essential for learning and improving of efficient technique(Bartlett, 1999)

\section{Methods}

Subjects: High level player (age $32 \mathrm{y}$; height $181 \mathrm{~cm}$; mass $75 \mathrm{~kg}$ ) volunteered in this study and he was informed about protocol procedures. The protocol was constructed in accordance with the Declaration of Helsinki.

Table (1):

Descriptive values Mean \pm SD of force and momentum for Center of gravity and hitting hand

\begin{tabular}{|c|c|c|c|c|c|c|c|c|}
\hline \multirow{2}{*}{ Instant } & \multicolumn{2}{|c|}{ CG_mntm } & \multicolumn{2}{|c|}{ Hand_mntm } & \multicolumn{2}{|c|}{ CG_F } & \multicolumn{2}{|c|}{ Hand_F } \\
\hline & Mean & SD & Mean & SD & Mean & SD & Mean & SD \\
\hline End of back swing & 43.33 & 7.43 & 1.24 & 0.37 & 502.37 & 217.94 & 14.92 & 2.66 \\
\hline Start of takeoff & 60.18 & 8.30 & 1.47 & 1.19 & 1111.78 & 456.27 & 40.46 & 34.80 \\
\hline End of takeoff & 94.55 & 12.77 & 3.97 & 1.75 & 1392.62 & 384.59 & 131.71 & 69.52 \\
\hline Ball impact & 41.70 & 14.98 & 4.78 & 2.34 & 1266.99 & 658.30 & 119.47 & 58.27 \\
\hline Ground contact & 72.76 & 22.01 & 2.72 & 1.16 & 823.39 & 96.87 & 19.66 & 16.22 \\
\hline
\end{tabular}

Figure (1):

shows the selected five instant

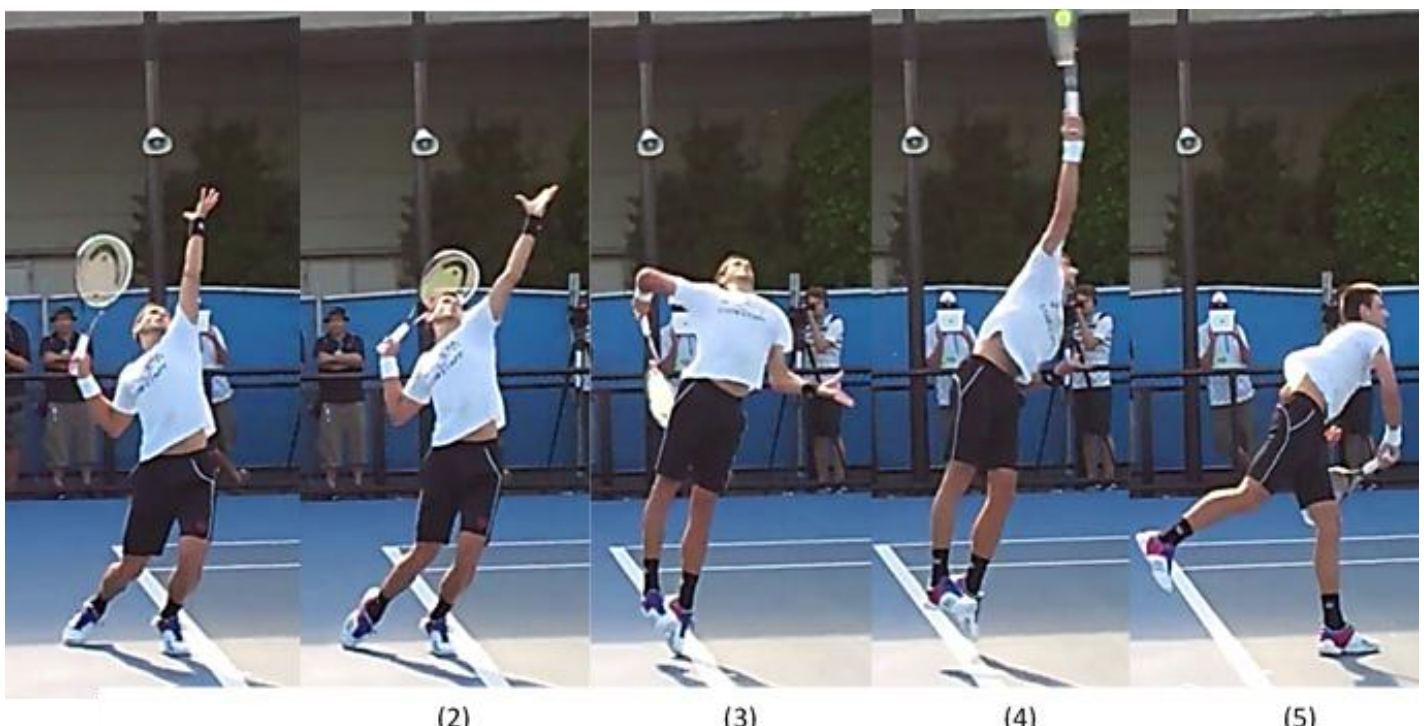

Procedures: after the player warm up he had five trials before recording. Six trials were captured using two synchronize camera (Basler $100 \mathrm{~Hz}$ ).the best successful three trials were selected for 3D motion analysis using (Winanalyze v2.0).

Statistical analyses:

Descriptive values (Mean \pm SD) were obtained using IBM SPSS Statistics 21 software

\section{Results:}

Table (1): presents the descriptive values (Mean \pm SD) of force and momentum for Center of gravity and hitting hand. Figure (2) shows the momentum curve for Center of gravity and hitting hand. Figure (3) shows the force curve for Center of gravity and hitting hand. Table (2) presents Mean \pm SD for phase time. Figure (4) shows the duration time of performance phase 
Figure (2):

CG and hitting Hand momentum curve at the five instant

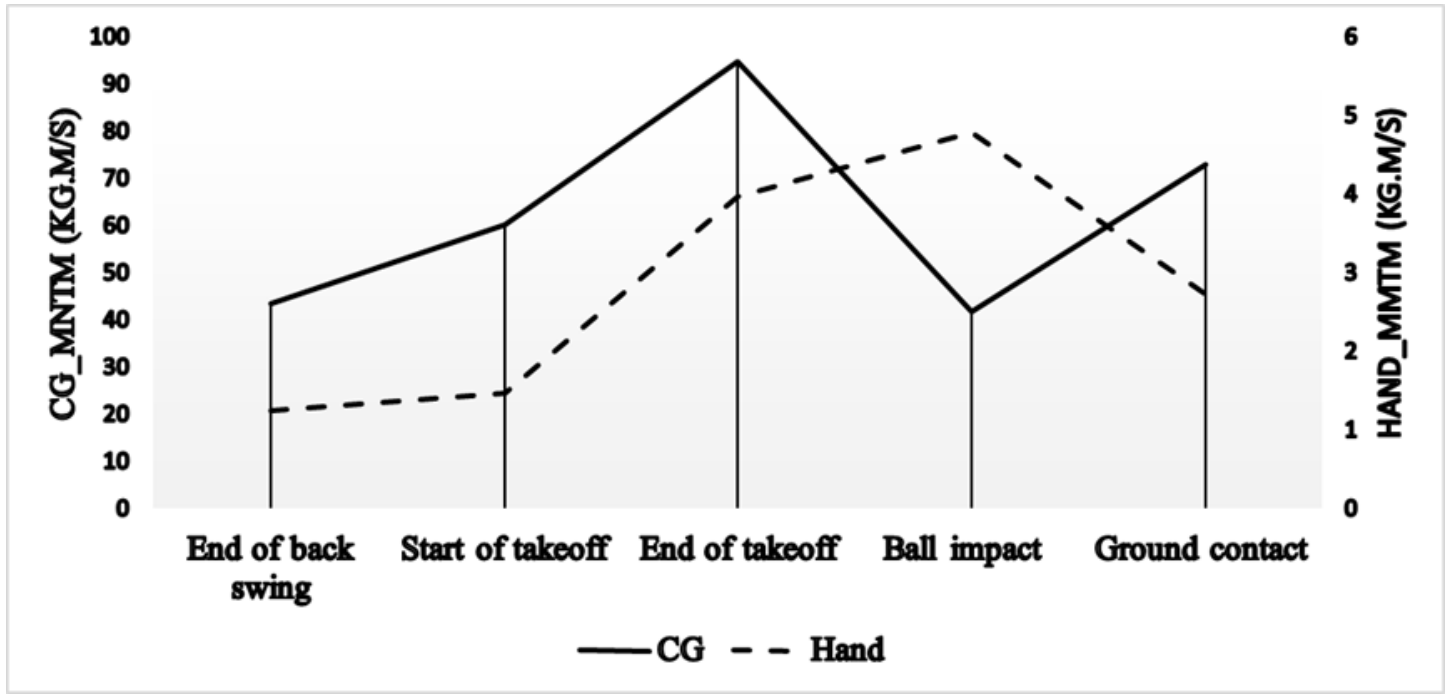

Figure (3):

CG and hitting Hand force curve at the five instant

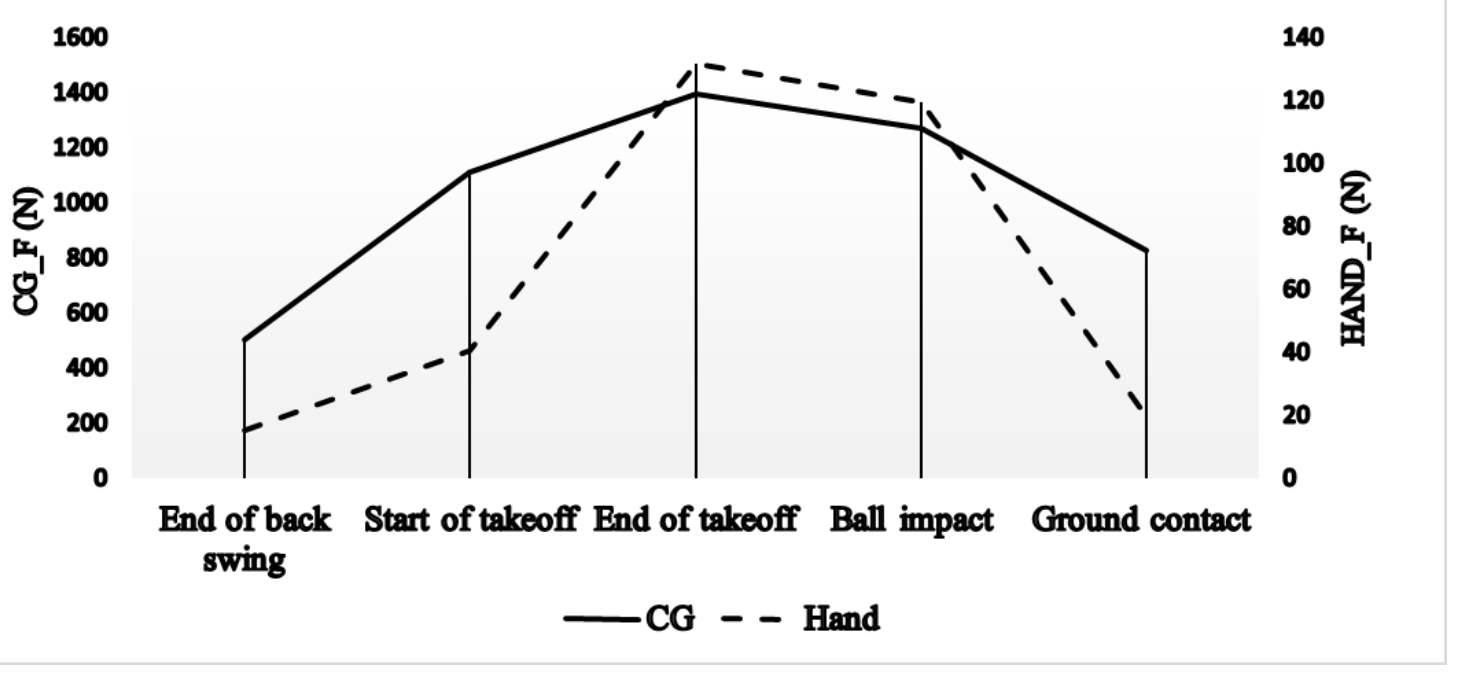

Table (2):

the Mean and \pm SD of phases time

\begin{tabular}{|c|l|c|}
\hline phases & Mean & SD \\
\hline Forward swing & 0.100 & 0.010 \\
\hline Leg drive & 0.147 & 0.021 \\
\hline Impact & 0.073 & 0.006 \\
\hline Landing & 0.130 & 0.020 \\
\hline
\end{tabular}


Figure (4)

the duration time of performance phase

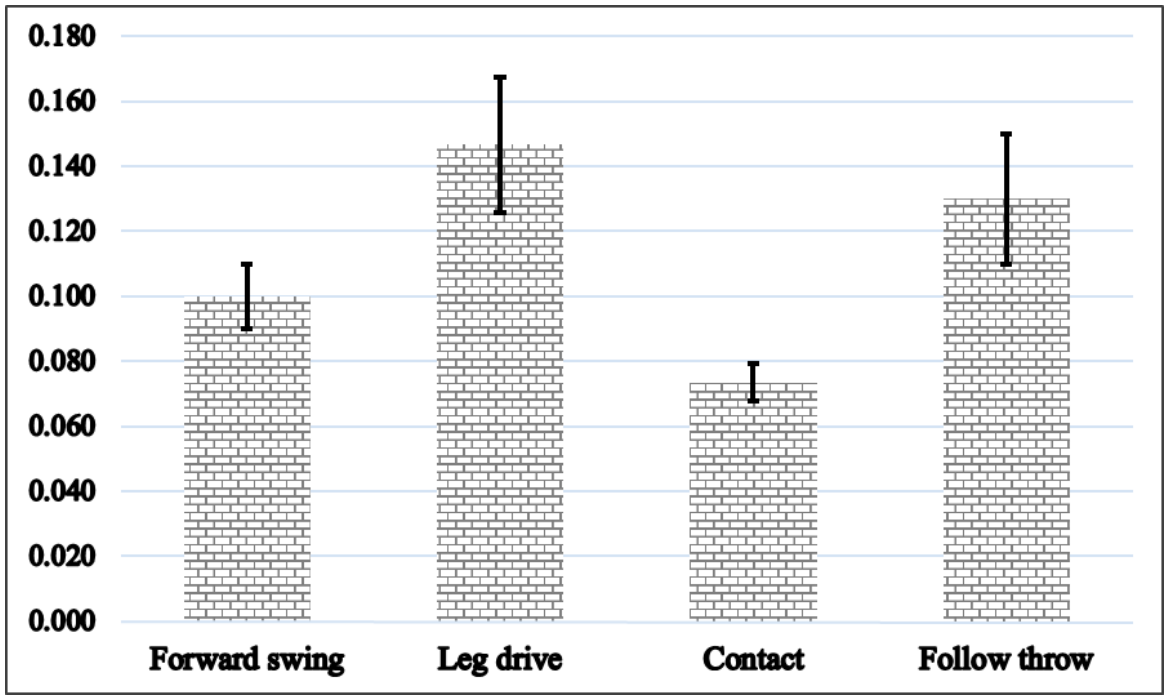

\section{Discussion:}

Many studies of tennis serving have been done, focusing on the upper-limb movements (B. Elliott, Fleisig, Nicholls, \& Escamilia, 2003; M. Reid, Elliott, \& Alderson, 2007), or the patterns of the lower-limb(Girard, Micallef, \& Millet, 2005), or the function of trunk(Chow, Park, \& Tillman, 2009; Chow, Shim, \& Lim, 2003).This study showed the trajectory of center of gravity and hitting hand motion and how the momentum and force transfer from the whole body to the hitting hand; in addition to temporal analysis for five instant and four phases. Table (1) and figure (2) showed the momentum curve for Center of gravity and hitting hand. All instant have a same curve except the instant of contact with the ball. Center of gravity curve is down at this instant and this is occurs because the body does not contact with the ground and becomes a projectile in the flight phase and it doesn't gain any momentum except that momentum obtained from loading stage forces created by a forceful leg drive. Therefore, maximizing the leg motion will help produce a consistent leg drive that can enhance the performance. (M. S. Kovacs \& T. S. Ellenbecker, 2011). While the hitting hand still have a momentum from foreword swing. It is well known that the legs require some degree of knee flexion during the preparation phase not only to decrease the loading in upper limbs segments(B. Elliott et al., 2003); but also to assist players in driving the racquet down behind, and away from the back (putting shoulder

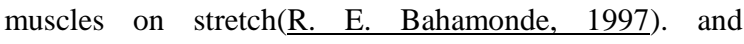
increasing the trajectory of the racquet prior to impact ( $\underline{\mathrm{b}}$. C. Elliott, t. Marsh,b. Blanksby, 1986). It should be assumed that the action of the lower extremities enhances the trunk and upper-arm rotations and facilitates the downward racquet motion and( $\underline{\text { R. E. Bahamonde, 1997) }}$ has shown that leg drive and trunk rotations produce a forced external (away from the direction of the serve) rotation of the upper arm, resulting in the stretch of the internal rotators muscles. On movement reversal, these stretched muscles are creating a higher speed of rotation of the hitting arm and consequently a higher post impact ball speed (Bruce Elliott, 2003).The purpose of the wind-up is to store elastic potential energy or strain energy. Strain energy occurs because the player muscles are stretched, the elastic recoil of the athlete's muscles convert the strain energy into kinetic energy, thus generating a tremendous amount of force and momentum(Carr, 1997). $(\boldsymbol{P}=\boldsymbol{m} \boldsymbol{V})$ therefore to produce an effect of momentum during the serve, since mass is constant, the velocity of the body must be increased. This is occur by the extension of the hitting arm along with the trunk and moving the Center of gravity forward to produce a good momentum in striking the tennis ball, this intern with the hitting striking the ball turns it into a force, this force is equal to the momentum of the body. In the tennis serve force is important to hit the ball $(\boldsymbol{F}=\boldsymbol{m a})$ the body mass is constant. So if the server has a great amount of acceleration then there will be a great amount of force according to this equation maximum acceleration is achieved by moving the whole body in a forward motion with continuity and timing. There are really no extraneous movements because most movements like the extension of the legs, trunk, hitting arm and the moving forward of the body are all extended to create greater body momentum. Therefore, the large involvement of the lower extremities in the tennis serve reinforces the importance of their strength and flexibility training to 
improve explosive power, speed, and endurance(M. Reid, A. Quinn, M. crespo, 2003)

Table (1) and figure (3) showed the force curve for Center of gravity and hand strike and all instant have a same curve with the different of mass between hand and whole body that is explain the force production from whole body segments to create more power and momentum due to them shifting their body weight forward allowing connection with the ball and allowing a smoother follow through

Timing is a very important factor and it should be considered during training process, because it is probably the hardest thing to get down to create maximum acceleration and effective motion. Figure (4) shows the duration of every phase. The largest time $(0.147 \pm 0.021)$ was in the leg drive phase which starts from push up to leave the ground; the player tries to generate force from pushing. (B. C. Elliott, Marshall, \& Noffal, 1995). The optimal leg drive mechanics and internal rotation, arm flexibility are critical for efficiency and velocity. Maximizing leg motion can produce a consistent leg drive that may enhance shoulder rotation and more efficient serves. (M. Kovacs \& T. Ellenbecker, 2011).

Second large time $(0.130 \pm 0.020)$ was in the follow throw phase which starts from the ball impact to touch the ground. The follow-through is a very important phase in the the tennis serve, requiring deceleration the eccentric loads in both the upper and lower body Continued glen humeral internal rotation and forearm pronation occur during the acceleration stage and continue after ball contact during deceleration. (M. Kovacs \& T. Ellenbecker, $\underline{2011)}$.

The third time $(0.100 \pm 0.010)$ was in the Forward swing which starts from the first noticeable movement that takes place during this second transition of the forward swing is internal rotation of the racket arm shoulder. Internal rotation, or medial rotation, is the inward turning of the upper arm in toward your body, however, with the racket arm in the drop position, internal rotation is more correctly defined as the upward and then forward rotation of the upper arm at the shoulder joint. The radius of hitting arm should lengthened before hitting to produce great serve the body's radius is lengthened in time corresponds with the fall of the ball to hit at a certain point. In the current study the minimum time $(0.073 \pm 0.006)$ was in the contact phase which starts from leaving the ground to hit the ball. Therefore it must done in less time.

\section{Conclusion:}

This study showed the force curve for center of gravity and hitting hand have a same path but it is a little different in the momentum curve at the impact instant. This is suggests that combining of balance and coordination exercises may help to maintain the Center of gravity force trajectory in the same path with the hitting hand which gives an advantage for success serve. The takeoff within minimum time helps to increase the velocity of the Center of gravity and doesn't loss a generated force from the body to the hitting hand

\section{References:}

1. Bahamonde, R. (2000). Changes in angular momentum during the tennis serve. Journal of Sports Sciences, 18(8), 579-592.

2. Bahamonde, R. E. (1997). JOINT POWER PRODUCTION DURING FLAT AND SLICE TENNIS SERVES Paper presented at the 15th International Symposiumon Biomechanics in Sports .

3. Bartlett, R. (1999). Sports biomechanics reducing injury and improving performance, from http://search.ebscohost.com/login.aspx?direct=true\& scope $=$ site $\& d b=$ nlebk $\& d b=$ nlabk $\& A N=76841$

4. Blazevich, A. (2012). Sports biomechanics : thebasics : optimising human performance. London: Bloomsbury.

5. Carr, G. A. (1997). Mechanics of sport : a practitioner's guide. Champaign, Ill.: Human Kinetics.

6. Cauraugh, J. H. (1990). TENNIS SERVING VELOCITY AND ACCURACY. PMS Perceptual and Motor Skills, 70 .(3)

7. Chow, J. W., Park, S. A., \& Tillman, M. D. (2009). Lower trunk kinematics and muscle activity during different types of tennis serves. Sports Med Arthrosc Rehabil Ther Technol, 1(1), 24. doi: 10.1186/17582555-1-24

8. Chow, J. W., Shim, J. H., \& Lim, Y .T. (2003). Lower trunk muscle activity during the tennis serve. J Sci Med Sport, 6(4), 512-518 .

9. De Subijana, C. L., \& Navarro, E. (2010). Kinetic energy transfer during the Tennis serve. Biol. Sport Biology of Sport, 27(4), 279-287.

10. Elliott, B. (2003 .(Biomechanics of advanced tennis. London: ITF.

11. Elliott, B., Fleisig, G., Nicholls, R., \& Escamilia, R. (2003). Technique effects on upper limb loading in the tennis serve. J Sci Med Sport, 6(1), 76-87 .

12. Elliott, b. C., t. Marsh,b. Blanksby. (1986). A ThreeDimensional Cinematographic Analysis of the Tennis Serve. JAB, 2(4), 260-271 .

13. Girard, O., Micallef, J. P., \& Millet, G. P. (2005). Lower-limb activity during the power serve in tennis: effects of performance level. Med Sci Sports Exerc, 37(6), 1021-102 .9 
14. Kleshnev, V. (2007). TEMPORAL ANALYSIS OF HITTING CYCLE IN ROWING. Paper presented at the XXV ISBS Symposium, Ouro Preto - Brazil

15. Knudson, D., \& Morrison, C. (2000). Visual Ratings of the Vertical Jump are Weakly Correlated with Perceptual Style. Journal of human movement studies., 39, 33-44 .

16. Kovacs, M., Chandler, W. B., \& Chandler, T. J. (2007). Tennis training enhancing on-court performance, from http://www.contentreserve.com/TitleInfo.asp? $\mathrm{ID}=\{1$ 885E4FA-C511-410D-9199-

4DB48C8E58FF $\&$ Format $=50$

17. Kovacs ,M., \& Ellenbecker, T. (2011). An 8-Stage Model for Evaluating the Tennis Serve: Implications for Performance Enhancement and Injury Prevention. Sports Health, 3(6), 504-513.
18. Kovacs, M. S., \& Ellenbecker, T. S. (2011). A performance evaluation of the tennis serve: Implications for strength, speed, power, and flexibility training. Strength Cond. J. Strength and Conditioning Journal, 33(4), 22-30 .

19. Kuo-ChengLo, L.-H. W., Chia-Ching Wu and FongChinSu. (2004). Kinematics of Lower Extremity in Tennis Flat and Spin Serve. Journal of Medical and Biological Engineering, 24(4 .(

20. Reid, M., A. Quinn, M. crespo. (2003). Increasing power in tennis. In: Strength and Conditioning for Tennis. Paper presented at the ITF Ltd, London .

21. Reid, M., Elliott, B., \& Alderson, J .(2007) . Shoulder joint loading in the high performance flat and kick tennis serves. Br J Sports Med, 41(12), 884-889; discussion 889 . doi: 10.1136/bjsm.2007.036657 
